\title{
Formulation and Characterization of Albumin/Glycine Microspheres as Oral Delivery System for Resveratrol
}

\author{
Kwame G Yeboah ${ }^{1 *}$ and Aladin Siddig ${ }^{2}$ \\ ${ }^{1}$ College of Pharmacy, Harding University, Searcy, USA \\ ${ }^{2}$ School of Pharmacy, University of Charleston, USA \\ *Corresponding Author: Kwame G Yeboah, College of Pharmacy, Harding \\ University, Searcy, USA.
}

Received: September 22, 2020

Published: October 08, 2020

(C) All rights are reserved by Kwame G Yeboah and Aladin Siddig.

\begin{abstract}
Purpose: Resveratrol has been reported to have beneficial effect against many diseases and has multi-spectrum therapeutic applications. However, oral delivery in its free form has short biological half-life with the consequent reduced bioavailability of only one percent. As such, the search continues for a polymer matrix that will be ideal for the protection of the drug and other compounds associated with food system from the conditions encountered in the gastrointestinal tract and to increase therapeutic efficiency.

Purpose of the Study: The purpose of this project therefore, was to evaluate the novel strategy of using albumin and glycine copolymer system to prepare microspheres capable of protecting the Resveratrol and meeting the sustained release specifications for increased bioavailability.

Method: Microspheres containing Resveratrol were prepared using BSA, Glycine and a mixture of BSA/Glycine polymer matrices. The comparative physicochemical characteristics such as particle sizes, zeta potential, drug release, and residual concentration of glutaraldehyde after the crosslinking process for six formulations were evaluated.

Results: The study showed that all the physicochemical characteristics of the selected BSA/Glycine microspheres were ideal for delivery of the resveratrol drug by the oral route. The drug substance was protected at extremely high temperature and oxygen stress. In addition, the residual glutaraldehyde concentrations were below the levels reported to be toxic for peroral delivery. Secondary, a novel means of neutralizing any excess glutaraldehyde by the use of sodium bisulfite was successful incorporated into the formulation.

Conclusion: It was concluded from the results of the study that BSA/Glycine copolymers can be used to produce microspheres with the required specifications for the oral delivery of bioactive compounds such as resveratrol.
\end{abstract}

Keywords: Resveratrol; Glutaraldehyde; Crosslinking; Glycine; Bioactive Compounds; Microspheres

\section{Abbreviations}

AMA1: Apical Membrane Antigen 1; BSA: Bovine Serum Albumin; DNA: Deoxyribonucleic Acid; Gly: Glycine; kDa: kilodalton; mV: millivolts; PBS: Phosphate Buffered Saline; PDI: Polydispersity Index; RES: Resveratrol; RNA: Ribonucleic Acid; UV/Vis: Ultraviolet/Visible; w/v: Weight-by-Volume; w/w: Weight-by-Weight

\section{Introduction}

With the increasing prevalence of chronic diseases and the associated long-term treatment schedules with possible adverse side effects, there has been a shift of interest to the use of natural compounds with wide accessibility and few side effects for the prevention and control of these diseases [1]. One of such compounds is Resveratrol (3,5,40-trihydroxy-trans-stilbene), a natural nonfla- 
vonoid polyphenol phytoalexin present in a wide variety of plants species, particularly in grapes, blueberries, and peanuts [2]. Resveratrol has been found to have beneficial effects against many diseases and has multi-spectrum therapeutic application as anticancer, immunomodulatory, antiangiogenic, cardioprotective and anti-oxidant agent [3].

However, oral administration of resveratrol (RES) in its free form has short biological half-life of only 10 minutes [1]. and the consequent reduced bioavailability of less than $1 \%$ as results of its poor aqueous solubility of less than $0.03 \mathrm{mg} / \mathrm{ml}$ [4], its isomerization or degradation by light, elevated temperatures and $\mathrm{pH}$ changes, high levels of auto-oxidation as well as its rapid and extensive pre-systemic metabolism and elimination [5]. This low bioavailability has been reported to limit the accumulation of the drug at the appropriate concentrations in the target tissues required for therapeutic effectiveness [6]. Meanwhile, increase in dose to offset these numerous limitations, has been reported to be associated with mild to moderate gastrointestinal symptoms [7]. Moreover, the gut microbiota hydrogenates the stable trans isomer, thus making it biologically less stable by oral administration [8,9]. These factors have put a limitation on the use of resveratrol in food and oral pharmaceutical products. It has therefore, become necessary to investigate novel formulation approaches to increase the bioavailability by increasing its solubility in water, stabilize and protect the drug from degradation, to achieve a sustained release to avoid dosing discrepancies and to target resveratrol to specific locations. Microencapsulation technique have been used to improve the bioavailability of many bioactive drug molecules including resveratrol by protecting them against unfavourable conditions such as light, oxidative enzymes, and other metabolic interference [10]. Furthermore, many biocompatible and biodegradable macromolecular materials have been used to encapsulate different types of poorly soluble drug molecules, including natural compounds like flavonoids and other bioactive substances to form micro/nanoparticles with targeted and/or controlled/sustained drug release abilities, resulting in their enhanced solubility, prolonged intestinal retention time, and improved oral absorption [11]. Moreover, the sustained release characteristics of encapsulation helps to increase the residence time for its action in the plasma and resulting better bioavailability [10].

Microspheres have become the major oral drug delivery system because of its extended release characteristic and the capacity to protect drug compounds from exposure to and degradation by gastric fluid $[12,13]$. From a physicochemical perspective, the micro- and nano-size range with the associated large surface area, and variable polymer composition that can be modulated to obtain defined behaviors are some of the characteristics that make microspheres suitable for oral delivery $[12,14]$.

Several biodegradable natural and synthetic polymers have been used to prepare microsphere. The natural polymers are mostly made of proteins (albumin, globulin, gelatin, collagen, and casein) and polysaccharides (starch, cellulose, and chitosan) [15]. Proteins are amphiphilic with strong binding to nutraceuticals like resveratrol through hydrophobic interactions and hydrogen bonding [16].

For this project, natural polymers such albumin (a protein) and glycine (an amino acid) were employed as co-polymers to prepare the microspheres because they are natural products of humans, readily available, relatively inexpensive and capable of a variety of chemical modifications [13].

Albumin microspheres have been extensively investigated in controlled release systems as vehicles for the delivery of therapeutic agents [12]. Human serum albumin has been reported to be very effective at improving the chemical stability of resveratrol [17]. Furthermore, $\alpha$-lactalbumin increased the solubility of resveratrol by as much as 32 times compared to the free drug [18]. Albumin has also been used as a carrier for targeting drugs to tumors and inflamed joints [13]. Intravenous administration of drugs coupled with albumin has been reported to improve the targeting efficiency of the formulation and the circulating half-lives of drugs have been reported to dramatically increase when conjugated to albumin [19]. Moreover, albumin has been extensively used in blocking buffers for solid-based immunoassay because of its ability to stabilize the antigenic and functional regions of the adsorbed proteins. This is done by saturating excess protein-binding sites on membranes and microplates [13]. The major exploitable features of albumin include is its biodegradation into natural products, its non-antigenicity and lack of toxicity [19,20].

Glycine and its compounds such as glycine betaine have been used extensively to protect bioactive compounds such as proteins from denaturation and deactivation by, for example, chaotropic compounds and elevated temperature $[13,21]$. It was used as a bulking agent in a spray-freeze drying in dry powder inhaler formulation of low aqueous solubility compounds such as salbutamol 
(albuterol) and budesonide to prevent aggregation and to enhance the dispersibility of the powders [22]. It has been reported that the addition of glycine or glycylglycine to a formulation containing the apical membrane antigen 1 (AMA1) of malaria parasites prevented the loss of potency of the vaccine in clinical trials [23]. Glycine has been used to optimize the recovery and longevity of active enzymes in vitro and as stabilizer to prevent inactivation of enzymes in storage media [24]. In addition, glycine is used to provide support during freeze-drying to prevent the macroscopic collapse of the final products in a number of formulations [25]. The amino acid has also been used in formulations to prevent the crosslinking of active pharmaceutical products. For instance, studies have demonstrated that when glycine and citric acid are present in some gelatin capsule formulations, pellicle formation or crosslinking of the capsule gelatin is prevented [26].

However, for these protein polymers to serve efficiently as controlled-release matrix, there is the need for modification to create a structure more stable than the readily soluble polar base forms that crumble in aqueous environment and by $\mathrm{pH}$ inactivation [27]. The most popular and efficient method for protein modification to achieve sustain-release properties is the crosslinking process that chemically joins two or more molecules of the protein polymer by covalent bonds. Furthermore, the rates at which the microspheres will degrade in the body have been found to be dependent on the degree of crosslinking of the albumin and glycine polymer matrix [12]. Among the many available protein crosslinking agents, glutaraldehyde is the most popular. It has found application in various fields such as histochemistry, microscopy, biochemical and pharmaceutical sciences [28]. Glutaraldehyde is commercially available at low cost and is more efficient than other aldehydes in generating thermally and chemically stable protein crosslinks [28]. In spite of the efficiency in crosslinking, free glutaraldehyde has been classified as toxic and repeated exposure to airborne droplets is reported to cause irritations. It is also widely used as a tissue fixative for histology and has been suggested that the same properties that make it a good tissue fixative may cause adverse effects clinically [29]. It is, therefore, necessary to monitor and neutralized any amount of the reagent not used in the crosslinking process during the formulation development.

\section{Purpose of the Study}

The purpose of the present study, therefore, was to prepare Bovine Serum Albumin (BSA)/Glycine (Gly) microspheres loaded with
Resveratrol (RES) using the Spray Drying technique. The amount of glutaraldehyde remaining after the crosslinking were determined and neutralized. The prepared microspheres were evaluated for their physicochemical characteristics such as particle size, encapsulation efficiency and zeta potential as well as the solid-state stability and in vitro drug release.

\section{Materials and Methods}

Materials

\section{Chemicals}

Bovine Serum Albumin (Fraction V, DNAase, RNAase, and Proteinase-free), Glycine, sterile de-ionized water, Phosphate Buffered Saline (1x PBS) pH 7.0, Glutaraldehyde (50\%), Phenol, and Perchloric acid (70\%) and sodium bisulfite were purchased from Fischer Scientific (Hanover Park, IL). Resveratrol (RES, $\geq 98 \%$ ) was purchased from Sigma-Aldrich Chemicals (Saint Louis, MO).

\section{Equipment}

The Buchi B-290 Mini Spray Dryer and M560 Melting Point apparatus were obtained from Buchi Corporation (Newcastle, DE). The Malvern Zetasizer Nano ZS (used for the zeta-potential analysis) was obtained from Malvern Instruments (Worca, UK). The Horiba LA920 laser scattering particle size distribution analyzer (used for the microsphere particle size analysis) was obtained from Horiba Instruments Incorporated (Irvine, CA). UV/Vis Spectrophotometer Shimadzu UV-1650PC (Beckman, Fullerton, CA) was used to determine drug concentration. The Distek Dissolution Apparatus system model 2100C, USP Dissolution apparatus 1, with rotating basket (used for the release studies) was obtained from Distek Inc. (North Brushwick, NJ).

\section{Methods}

\section{Preparation of microspheres}

Three sets of microspheres were prepared individually from Albumin (BSA), Glycine (Gly) and a mixture of equal amount of Albumin and Glycine (BSA/Gly). For each of these microsphere formulations, there were three sets of drug loading: the blank microspheres did not contain the resveratrol drug, the second and third sets of microspheres contained $1 \%$ and $10 \%$ resveratrol.

Formulation of polymer matrix by crosslinking with Glutaraldehyde

Two sets of five percent weight by volume $(\mathrm{w} / \mathrm{v})$ solutions of Albumin, Glycine and a mixture of equal amount (2.5\% each) of 
Albumin and Glycine were prepared in deionized water. Glutaraldehyde as crosslinking agent was mixed in the matrix solutions in concentrations of $0.5 \%$ and $1.0 \%$ volume-by-volume respectively. The solutions were kept in the dark at room temperature for 24 hours to allow polymerization.

Determination of used, and residual concentration of glutaraldehyde

The amount of glutaraldehyde used in the crosslinking process and residual concentration left after the crosslinking were determined by the phenol/perchloric acid method described by Furst and Banerjee and Boratynski and Zal $[29,30]$. Briefly, two hundred microliters sample of the polymer solutions containing the crosslinking glutaraldehyde reagents were collected at predetermined time points of $1,2,4,8,12$ and 24 hours during the crosslinking processes. These samples and a glutaraldehyde standard solution were mixed with $1 \mathrm{ml}$ of phenol reagent made of 40 microliters of $5 \%$ phenol in water in $10 \mathrm{ml}$ of $70 \%$ perchloric acid and incubated for 15 minutes. Aqueous solutions of glutaraldehyde produce yellow color when treated with phenol. Color intensity in absorbance was measured versus the phenol reagent at wavelength of $479 \mathrm{~nm}$ with a UV/Vis Spectrophotometer. The quantities of used at each time point and the amount remaining after the 24 hours of crosslinking were determined.

Preparation of Albumin and Glycine microspheres loaded with Resveratrol [13]

From the results of the glutaraldehyde crosslinking efficiency analysis, the $0.5 \% \mathrm{v} / \mathrm{v}$ glutaraldehyde crosslinking was used to prepare the microspheres. Two sets of microsphere formulations with $1 \%$ and $10 \%$ Resveratrol $(\mathrm{w} / \mathrm{w}$ ) loading were prepared by crosslinking a $5 \% \mathrm{w} / \mathrm{v}$ concentration each of albumin, glycine and a mixture of $2.5 \%(\mathrm{w} / \mathrm{v})$ each of albumin and glycine in aqueous solutions with the $0.5 \%$ volume-by-volume concentration of glutaraldehyde for 24 hours at room temperature. At the end of the crosslinking, the excess glutaraldehyde was removed by chemical deactivation using sodium bisulfite $[13,31]$. A weight of resveratrol drug powder equivalent to $1.0 \%$ and $10.0 \%$ with respect to the weights of the protein polymers in the matrixes were added to the crosslinked polymer suspensions and spray dried with the Bulchi B-290 mini spray dryer. The spray drying process was optimized at a setting of $5 \%$ and an inlet temperature of $110^{\circ} \mathrm{C}$ given an outlet temperature of $74^{\circ} \mathrm{C}$ and $60 \%$ aspirator with compression flow rate of 800 psi.

\section{Product yield}

The product yield - that is total amount of microspheres produced as a percentage of the total amount of all ingredients used was calculated by the following formula:

Product Yield \% = [Weight of microspheres obtained from spray dryer/Weight of the total amount of solids in the feed] X 100

Physico-chemical characterization of microspheres

\section{Measurement of particle size}

The particle sizes and size distribution of the microsphere formulations were measured using the Horiba IA920 laser scattering particle size distribution analyzer. Briefly, 1 milligram of microspheres were suspended in $3 \mathrm{ml}$ of Milli-Q ultra-pure water. The suspension was sonicated for 2 minutes before use.

\section{Measurement of zeta-potential}

The zeta-potential of the microspheres were measured using the Malvern Zetasizer Nano ZS series. 0.5 milligram of microspheres were suspended in 1 millimeter of Milli-Q ultra-pure water (pH 7.4) before use.

\section{Determination of encapsulation efficiency}

The encapsulation efficiency was to determine the efficiency of the microencapsulation method for drug loading. All the microspheres used were prepared with $0.5 \%$ glutaraldehyde crosslinking as a result of the prior crosslinking efficiency analysis. In this study, $10 \mathrm{mg}$ of dry microspheres samples from the six sets of drug-loaded microspheres - three sets of the $1 \%$ drug loading and three sets of the $10 \%$ drug loading each in the three protein polymer matrixes of albumin, glycine or albumin/glycine were added to 1 milliliter of deionized water containing $1 \%$ triton X-100. The dissolution was aided by gentle crushing of the microspheres with a glass rod. The test tubes were subjected to continuous sonication for 2 hours and placed on a rotary shaker in the darkness for 24 hours at room temperature. The solutions containing Resveratrol were filtered with $0.45 \mu \mathrm{m}$ filter paper and the samples were analyzed with Perking Elmer Spectrophotometer using 306 absorbance as reported by Trela and Waterhouse [32], and Camont., et al. [33]. The encapsulation efficiency was determined by the following equation:

Encapsulation Efficiency (\%) = [The total amount of Resveratrol determined in the Microspheres/ The theoretical amount of Resveratrol in the microspheres] $\mathrm{X} 100$ 


\section{Thermal stability studies}

Thermal stability was determined with Buchi M560 boiling point equipment. About $10 \mathrm{mg}$ of all the microsphere formulations including blanks and those containing Resveratrol were placed into boiling tubes, and introduced into the Buchi M560 equipment. The temperature rate was $5^{\circ} \mathrm{C}$ per minute $\left(5^{\circ} \mathrm{C} / \mathrm{min}\right)$. The boiling point for each sample was recorded.

\section{In vitro release profile and mechanism of drug release}

The in vitro drug release studies were performed with Distek Dissolution system Model 2100C USP Apparatus 1 with 40-mesh rotating baskets. The studies were carried out over 24 hours in the dark to prevent isomerization/degradation of the released drug molecules by light. 25 milligrams of the $1 \%$ and $10 \%$ drug-loaded microspheres were placed in dialysis membrane bags with molecular weight cutoff of 6000 - 8000 Daltons and flat width of 40 millimeters. The bags were soaked in double-distilled water for 12 hours before use. One milliliter of Phosphate Buffered Saline (PBS, $\mathrm{pH}$ 7.0) was added to each dialysis membrane bag and placed into $250 \mathrm{ml}$ of PBS (pH 7.0). The apparatus was set at $37^{\circ} \mathrm{C}$ and $140 \mathrm{rpm}$. Samples were collected at zero, 1, 4, 8, and 24 hours. Five milliliters samples were removed and the vessels were replenished with fresh $5 \mathrm{ml}$ of PBS ( $\mathrm{pH}$ 7.0) to maintain sink conditions. The samples were filtered through $0.45 \mu \mathrm{m}$ membrane filter and analyzed for Resveratrol at $306 \mathrm{~nm}$.

In order to determine mechanism of drug release from the microspheres, the data obtained from the in vitro release studies on the $1.0 \%$ and $10.0 \%$ drug-loaded BSA/Glycine copolymer microspheres were fitted into various kinetic models.

\section{Release under oxygen stress and storage temperatures}

About $20 \mathrm{mg}$ each of the six microsphere formulations were mixed thoroughly in $10 \mathrm{ml}$ of $5 \%$ and $10 \%$ solutions of hydrogen peroxide $\left(\mathrm{H}_{2} \mathrm{O}_{2}\right)$ and $10 \mathrm{ml}$ of blank deionized water. The suspensions were transferred into $15 \mathrm{ml}$ conical tubes and incubated at $4^{\circ} \mathrm{C}$ and $25^{\circ} \mathrm{C}$ in the dark for 24 hours. $1 \mathrm{ml}$ samples of the mixtures were withdrawn and analyzed for resveratrol concentration using UV-Vis Spectrophotometer at $306 \mathrm{~nm}$ wavelength.

\section{Statistical analysis}

Statistical analyses were carried out for all the individual studies reported in this project. All comparative analyses were made between the different formulations and the blank microspheres when applicable. Statistical significance was determined with the use of ANOVA between groups, and a $p$ value of less than 0.05 was considered statistically significant

\section{Results and Discussion}

Determination of the usage and residual glutaraldehyde concentration

The residual concentration per time was evaluated as a marker of the rate of usage of the glutaraldehyde in the crosslinking process. This was necessary to determine the toxic levels of glutaraldehyde and, therefore, the subsequent amount of reagent needed to neutralize the residue. Figure 1 shows the standard curve for determining the glutaraldehyde concentrations. The $\mathrm{R}^{2}$ value of 0.9984 indicates a good correlation between the absorbance and concentration of the crosslinking reagent.

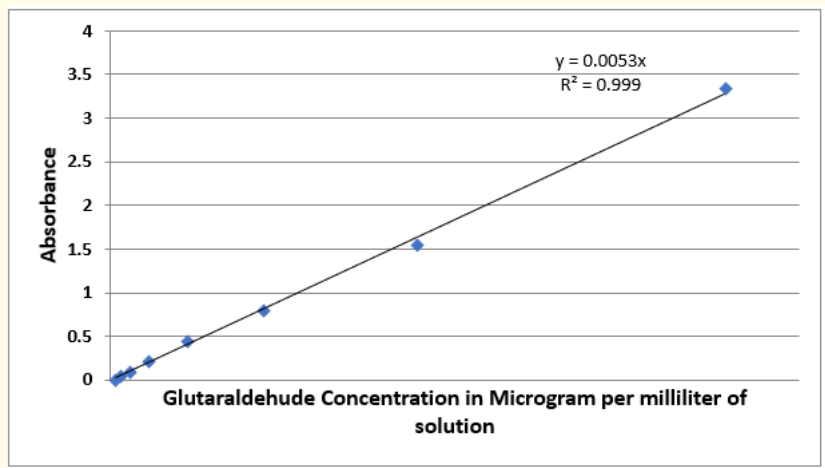

Figure 1: Glutaraldehyde concentration standard calibration curve.

The residual concentrations per time of glutaraldehyde in the polymer solutions during the crosslinking process has been shown in both table 1 and figure 2. The table 1 and figure 2a show the residual concentrations of all glutaraldehyde in all the polymer formulations for both the $0.5 \%$ and $1 \%$ of glutaraldehyde assessed in the study. The concentrations were found to be higher in the $1 \%$ crosslinking than the $0.5 \%$. This was expected as the $1 \%$ crosslinked matrices had the same concentrations of polymer material but double the concentration of glutaraldehyde. Moreover, the concentrations decreased with time as the reagent was being used up. For the $0.5 \%$ crosslinking, the concentration decreased gradually for the first 12 hours before plateauing for the all the formulations except the BSA/Glycine mixture polymer that plateaued after eight hours. For the $1 \%$ crosslinking, the glutaraldehyde concentration continued to the 24 hours without plateauing. This finding is very 
important for formulations containing drug compounds that may be reactive to glutaraldehyde to determine the optimum concentration needed and when it is necessary during the formulation process to have a good crosslinking and the residual amount of the reagent that would have significant effect on the drug, and be toxic as well. Figure $2 \mathrm{~b}$ shows the comparative concentrations for the BSA/Glycine polymer matrices selected for this project at the $0.5 \%$ and $1 \%$ crosslinking.

\begin{tabular}{|l|c|c|c|c|c|c|}
\hline \multirow{2}{*}{ Crosslinking Time in Hours } & \multicolumn{3}{|c|}{$\mathbf{0 . 5} \%$ Crosslinking (Conc in $\boldsymbol{\mu g} / \mathbf{m l}$ ) } & \multicolumn{3}{|c|}{$\mathbf{1 \%}$ Crosslinking (Conc in $\boldsymbol{\mu g} / \mathbf{m l}$ ) } \\
\cline { 2 - 7 } & $\mathbf{B S A}$ & Glycine & BSA/GLY & BSA & Glycine & BSA/GLY \\
\hline 1 & 53.8 & 52.8 & 42.7 & 122.6 & 125.9 & 121.3 \\
\hline 2 & 23.2 & 33.2 & 12.6 & 79.53 & 92.7 & 61.2 \\
\hline 4 & 9.6 & 13.6 & 0.6 & 44 & 58.4 & 34.4 \\
\hline 8 & 5.6 & 6.8 & 0.4 & 28 & 37.5 & 15.3 \\
\hline 12 & 3.6 & 3.8 & 0.27 & 13 & 16.9 & 7.9 \\
\hline 24 & 1.7 & 2.3 & 0.15 & 8.2 & 12.7 & 3.59 \\
\hline Percent Conc at 24 hours & 2.49 & 3.35 & 0.35 & 3.85 & 9.67 & 1.69 \\
\hline Percent Reduction in 24 hours & 97.59 & 69.75 & 99.65 & 96.15 & 90.33 & 98.31 \\
\hline
\end{tabular}

Table 1: Concentrations per time of glutaraldehyde in the polymer solutions during the crosslinking process.

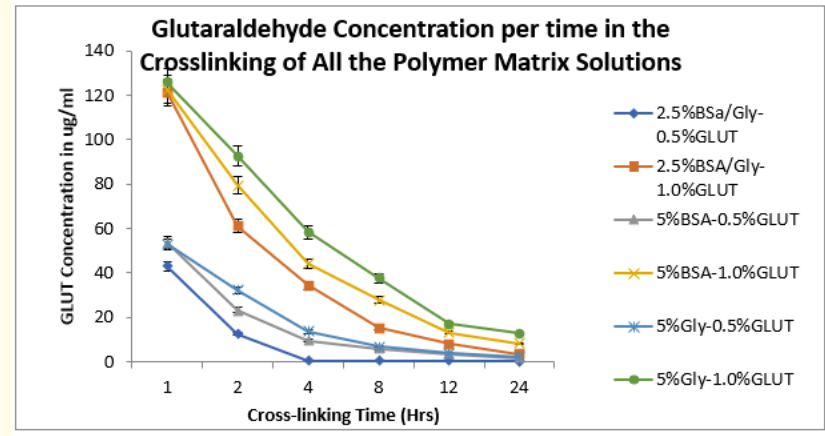

Figure 2a: Glutaraldehyde concentration per time in the various crosslinked polymer matrix solutions.

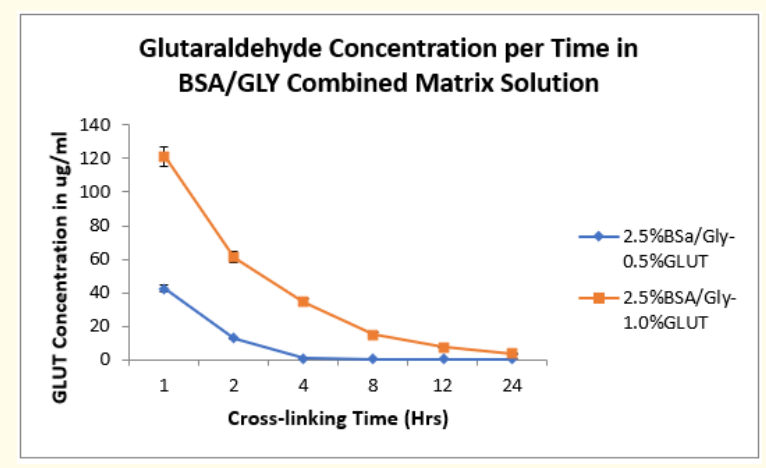

Figure 2b: Glutaraldehyde concentration per time in the BSA/ Glycine crosslinked copolymer matrix solutions.
Figure 3a-3c show the comparative concentrations and percent reduction of glutaraldehyde in the various polymer matrices. In all the polymer matrices investigated (Figure 3a), the residual concentrations after the 24 hours of crosslinking were significantly different from each other $(\mathrm{p}<0.02)$. In both the $0.5 \%$ and $1 \%$ crosslinking concentrations, there were very high percent reduction and lower concentrations of the reagent after the 24 hours in all the polymer matrices. As figure $3 \mathrm{~b}$ shows, there was a significant reduction in the BSA/Glycine copolymers than the BSA and Glycine alone in the $0.5 \%$ crosslinking group. The pattern was slightly different in the $1 \%$ crosslinking group (Figure $3 \mathrm{~b}$ ). In this group, even though the percent reduction was still higher in the BSA/Glycine copolymer than the BSA and Glycine polymers, the reduction between the BSA/Glycine copolymers and BSA was not significant ( $p$ $<0.12$ ), and the reduction in the BSA/Glycine and BSA were all significantly higher than the Glycine $(\mathrm{p}<0.05)$. In all the formulations tested, there were lower residual concentrations of the reagents in the $0.5 \%$ than the $1 \%$ crosslinking group. The final percent concentrations of glutaraldehyde in the BSA/Glycine copolymer were 0.35 and 1.69 of the starting concentrations for the $0.5 \%$ and $1 \%$ crosslinking respectively (Table 1). This brought the final formulation solution concentrations to $0.0021 \%$ and $0.0169 \%$ for the $0.5 \%$ and $1 \%$ crosslinking respectively. The final concentrations at 24 hours for the $0.5 \%$ and $1 \%$ crosslinking for the BSA/Glycine copolymers were 0.15 and 3.59 micrograms per milliliter of formulation solutions (Table 1). This works out to 1.5 and 35.9 parts per million 
solution concentrations. This shows a high reactivity of the glutaraldehyde towards proteins at the neutral $\mathrm{pH}$ of the crosslinking. The pattern shown here is similar to one reported by the authors in 2013 [13]. In 1996, Jordan and his co-workers determined that the $\mathrm{IC}_{50}$ of glutaraldehyde for bacterial inhibition was $17 \mathrm{ppm}$ [31], which is higher than the concentration reported in this study. In 2001, Ballantyne and Jordan [34] reported that aqueous solutions of $5 \%$ to $50 \%$ glutaraldehyde are of moderate acute oral toxicity but those of $2 \%$ or less were of no or slight toxicity [13]. Furthermore, Ballantine and Myers determined that the per oral $\mathrm{LD}_{50}$ range for $5 \%$ or higher glutaraldehyde solution in rats is between 0.88 to 3.25 milliliters per kilogram body weight. However, they found out that solutions between $1 \%$ and $2 \%$ were of slightly higher toxicity with the $\mathrm{LD}_{50}$ range between 3.34 to 12.30 milliliters per kilogram body weight $[13,35]$. The residual concentration of glutaraldehyde after 24 hours of crosslinking BSA/Glycine copolymers determined in this study were significantly less than that $(1.5 \mathrm{ppm}, 0.0021 \%$ and $35.9 \mathrm{ppm}, 0.0169 \%$ for the $0.5 \%$ and $1 \%$ crosslinking respectively). It can be inferred from this study that the excess glutaraldehyde present at the end of the 24 hours of crosslinking is safe for oral delivery. However, for the delivery of Resveratrol which is a bioactive compound associated with the food system, there is the need to neutralize or completely remove any excess glutaraldehyde that remains in the delivery system. One of the popular ways to neutralize and completely eliminate glutaraldehyde has been determined to be the use of sodium bisulfite (SBS) at a molar ration of 1:2.2 glutaraldehyde to SBS $[12,13,31]$. In this study, a ratio of 1:10 glutaraldehyde was used to neutralized the residual glutaraldehyde after the 24 hours crosslinking. This ratio has already been used by the authors in the formulation of Mycobacterium tuberculosis antigens in BSA polymer [12], and Riboflavin in BSA/Glycine copolymers [13] and found to be effective in neutralizing all residual glutaraldehyde thus making the crosslinked matrixes completely free of the reagent before the addition of the resveratrol.

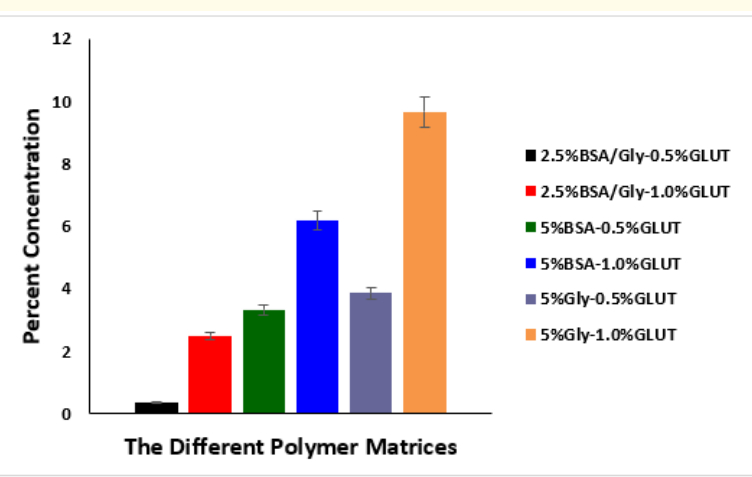

Figure 3a: Comparative percent residual/reduction relative to starting concentrations of glutaraldehyde in the BSA/Glycine, BSA, and Glycine polymer matrices after 24 hours of crosslinking.

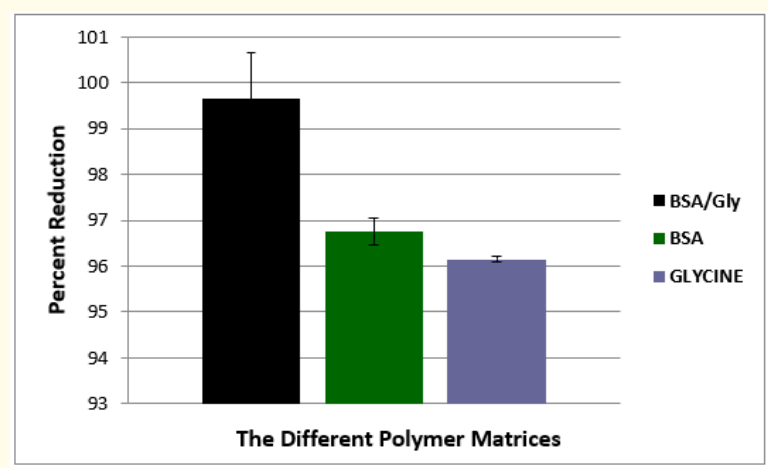

Figure 3b: Comparative percent reduction in the glutaraldehyde concentration after 24 hours for the $0.5 \%$ crosslinking.

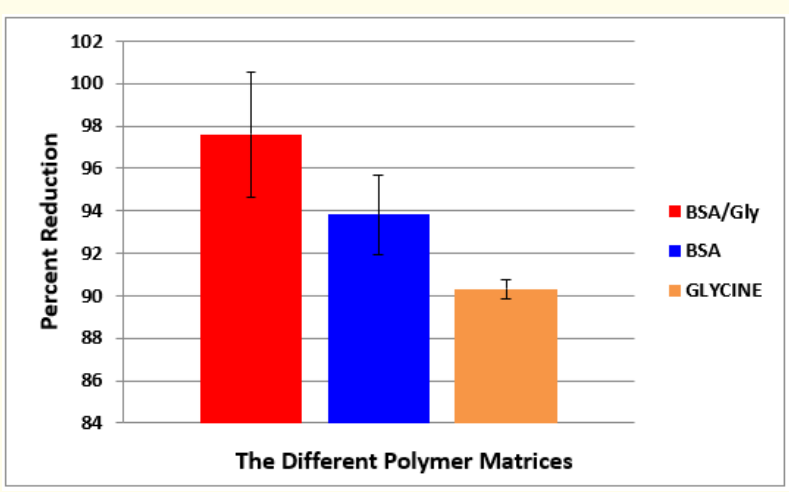

Figure 3c: Comparative percent reduction in the glutaraldehyde concentration after 24 hours for the $1.0 \%$ crosslinking.

\section{Physico-chemical characteristics of microspheres}

The physico-chemical characterization of drug products is useful in determining the efficiency of the product in delivering the active pharmaceutical ingredient in a manner that will enable the best therapeutic outcome.

The particle size and surface charge (as determined by the zeta potential) of the product particles are important for the suspendability in administrative liquids and stability of the formulations. They are also important in the interaction of the microspheres with the absorption surfaces of the intestines. The encapsulation efficiency and product yield are important in determining the efficiency of the formulation and production processes and are important in maximizing materials during scale-up to cut down cost. Furthermore, formulation and production of bioactive drug products involving the use of organic products such as protein polymers, and particularly by the use of the spray drying technique involves various chemical and physical stresses that can affect the stability of such drug compounds. Changes in the thermal properties of bioactive organic compounds such as resveratrol can indicate any the 
stability issues or otherwise of the formulations. Evaluation of the changes in the melting point for instance of such compounds is one of the several analytical methods that have been found to be useful in determining the stability of the active pharmaceutical ingredients in such formulations. The different formulations in the various polymer matrixes at $0.5 \% \mathrm{v} / \mathrm{v}$ glutaraldehyde crosslinking with the various loading loadings are shown in table $2 \mathrm{a}$.

\begin{tabular}{|l|c|c|}
\hline $\begin{array}{l}\text { Formulation } \\
\text { Number }\end{array}$ & $\begin{array}{c}\text { Polymer Type (5\% } \\
\text { w/v)/0.5\% Glutaraldehyde }\end{array}$ & Drug Loading \\
\hline Blank 1 & BSA/Glycine & No Drug \\
\hline Blank 2 & BSA & No Drug \\
\hline Blank 3 & Glycine & No Drug \\
\hline F1 & BSA/Glycine & $1.0 \%$ \\
\hline F2 & BSA & $1.0 \%$ \\
\hline F3 & Glycine & $1.0 \%$ \\
\hline F4 & BSA/Glycine & $10.0 \%$ \\
\hline F5 & BSA & $10.0 \%$ \\
\hline F6 & Glycine & $10.0 \%$ \\
\hline
\end{tabular}

Table 2a: The microsphere formulations with the types of polymer matrixes and drug loading.

Product yield, particle size, zeta potential, encapsulation efficiency and particle sizes of microsphere formulations

The basic physico-chemical characteristics of the $0.5 \%$ crosslinked microspheres from the three blank formulations of the individual polymer matrixes of BSA/Glycine, BSA, and Glycine as well as the formulations with drug loading are shown in table $2 \mathrm{~b}$.
Two different resveratrol drug loadings of $1.0 \%$ and $10.0 \%$ weightby-weight with respect to the amount of the different polymers were used for this study. The product yields were calculated as the amounts of microspheres recovered after the formulation and spray-drying processes as a percentage of the theoretical amounts of all ingredients used. As the table shows, the product yields for all the of formulations were very high and similar in each category of blanks, $1.0 \%$ and $10.0 \%$ drug loadings in all the matrices. The product yield for the blank microspheres were between an average of 70.3 to 77.7 percent and that of the $1.0 \%$ drug loading was between 73.8 to 79.7 percent. However, the $10.0 \%$ drug loaded microspheres had a relatively higher yield of between approximately 86.4 to 88.5 percent. Generally, with the same amount of polymer used, the product yield is theoretically dependent mostly on the extra weight of the drug loaded. The study, therefore, shows that the $1.0 \%$ drug loading did not lead to any appreciable increase in the product yield compared to the blank microspheres. But the extra and larger amount used in the $10.0 \%$ drug loading showed its effect in extra yield. However, the ranking of the product yield did not depend on the type of polymer matrix used. Whilst the BSA microspheres produced the second highest product yield in the blank (74.9\%) and $1.0 \%$ (79.7\%) drug loading, it had the highest yield of $88.5 \%$ in the $10.0 \%$ drug loading. The Glycine microspheres on the other hand showed a different pattern. It ranked highest with $77 \%$ product yield in the blank microspheres but came down to second and third with the $1.0 \%$ and $10.0 \%$ drug loading. The BSA/Glycine microspheres had the lowest yield with the blanks but became second with the $1.0 \%$ and $10.0 \%$ drug loading.

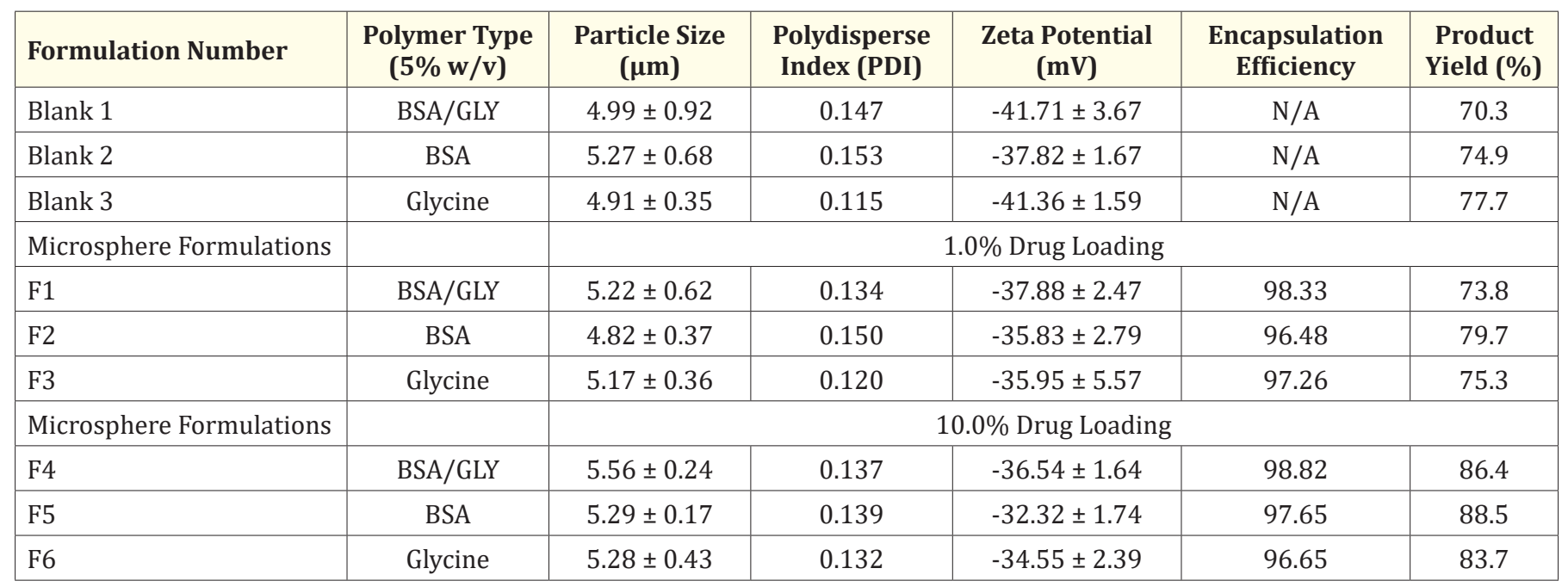

Table 2b: Particle size, zeta potential, encapsulation efficiency and product yield of microsphere formulations. 
Product yields determine the efficiency of the spray-drying production process and are important in scale-ups and estimating the quantities of drug and excipients needed. The yields of over 73 and 84 percent in the $1.0 \%$ and $10.0 \%$ drug loaded microspheres in this study show that the spray drying technique produced limited losses and can be used successfully in large scale industrial production. This may be due to the improved product collection in the modern spray-drying equipment. Galindo-Rodriquez and his coworkers in a comparative study, found that most other microencapsulation methods result in low yields because of difficulties associated with particle collection and purification particularly with small-sized microspheres [36]. It was reported that many microspheres with small sizes could not be recovered in the laboratory scale apparatus because of the inefficient deposition in the cyclone due to their low aerodynamic mass and are sucked away in the vacuum [37]. Furthermore, it has been determined that mass recovery of polydispersed microspheres in size range below 100 microns from all microencapsulation techniques are able to provide approximately $45 \%$ yield at best [38]. The high yield reported in this study from the typical laboratory-sized spray dryer with its reduced size and associated limited evaporation capacity, shows how the problem with low yield in spray drying has been addressed. Moreover, this problem is taken care of during industrial scale-up by the introduction of dry towers that are several meters in height.

The encapsulation efficiencies as shown in table $2 \mathrm{~b}$, were from 97.26 to 98.82 percent. The data shows that the encapsulation efficiency was very high with the formulation process. The efficiency interestingly was not affected by the increasing drug loading nor by the type of polymer matrices. These findings are similar to one reported by other investigators. Cho and his co-workers obtained encapsulation efficiencies between 97 to 99.5 percent with resveratrol in chitosan microspheres [39]. Similarly, Das and Ng reported an over $97.7 \%$ encapsulation efficiency of resveratrol in calciumpectinate microspheres [40]. The high encapsulation efficiency of resveratrol in microparticles is reported to be due to the drug's low solubility in water. As such it remains mostly undissolved with little losses during the formulation and crosslinking processes [41].

The data (Table 2b) show that the particle sizes of all the formulations were around 5 microns and the and Polydispersity index (PDI) were below 0.2. Furthermore, there was no significant change in the particle sizes with formulation and drug loading. Similarly, the sizes did not depend on polymer matrixes. Particle sizes of microspheres have been reported to depend mostly on the concentrations of the polymers in the primary suspension [42-44]. This means that the extra material associated with increased drug loading from the blank through the $1.0 \%$ to $10.0 \%$ did not have any significant effect on the droplet sizes generated during the spraying that led to the particle sizes. As the data in table $2 \mathrm{~b}$ show, the particle sizes for all the formulations were below 10 microns and ranging from 4.82 to 5.56 microns. These size range has been found to be ideal for oral delivery. In another study, Norris and Sinko [45] reported that microspheres with diameter of below 10 microns have the advantage of being more easily able to penetrate the mucus layer to reach the apical membrane of the epithelium layer of the absorption surfaces in the gastrointestinal tract. The microspheres produced in this study is therefore, ideal for oral absorption.

Polydispersity Index (PDI) is basically a representation of the distribution of sizes within a given formulation sample. It is used to describe the degree of non-uniformity of the size distribution or the heterogeneity of the particle sizes in a sample. The numerical value of PDI ranges from 0.0 (for perfectly uniform sample with respect to particles size - monodisperse) to 1.0 (for a highly polydisperse sample with multiple particle populations) [46]. The PDI obtained from all the formulations were between 0.115 to 0.153 (Table $2 \mathrm{~b}$ ). This shows a fairly uniform or narrow range of the particle sizes in the formulation and therefore, the homogeneity of the sizes of the particles. This is because, the values of 0.2 and below are reported to be the most commonly acceptable in practice for polymer-based microparticle materials [46]. The low PDI is an indication of the effectiveness of the spray drying equipment in creating uniform droplet sizes in the spraying phase of the process and its ability to prevent aggregation of the formed particles after the drying phase.

The Zeta potentials of all the formulations were over $-30 \mathrm{mV}$ (Table $2 \mathrm{~b}$ ). The zeta potentials were mostly due to the encapsulating polymer matrix because of the high values in the blank microspheres. The data show that the surface charges were moderated and reduced by addition of the resveratrol drug. However, values of over $-30 \mathrm{mV}$ is very good for the suspension stability of the formulations. It is reported that the higher the absolute value of zeta potential, the greater the repulsive forces between the particles thus limiting deflocculation and instability of the drug suspensions [12]. It was also reported by Ahsan and co-workers that the surface charge of microparticles has a significant effect on its uptake by cells. Their report further stated that the greater the potential 
from zero or the greater the absolute value, the more effective is the uptake [47].

\section{Thermal stability}

The comparative melting points of the resveratrol-loaded microspheres and the blank counterparts in the different polymer matrixes and their respective drug loading are shown in table 3 and figure 4 . The data show higher or comparable melting points for all the formulations to that of pure resveratrol which has a melting point of about $254^{\circ} \mathrm{C}$. The melting point of BSA was the highest of $296.7^{\circ} \mathrm{C}, 294.35^{\circ} \mathrm{C}$ and $301.14^{\circ} \mathrm{C}$ for the blank, $1.0 \%$ and $10.0 \%$ drug-loaded microspheres respectively. This may be because BSA has a large molecular weight of $66.5 \mathrm{kDa}$ and made up of 583 amino acids. Interestingly, there was no significant change in the melting point of the BSA microspheres with the addition of resveratrol drug. This shows that the quantity $(1.0 \%$ and $10.0 \% \mathrm{w} / \mathrm{w})$ of drug added to the large molecular weight albumin did not affect the energy needed to melt that quantity of material. However, and as expected, the melting points of the glycine formulations were $253.37^{\circ} \mathrm{C}, 253.75^{\circ} \mathrm{C}$ and $250.78^{\circ} \mathrm{C}$ for the blank, $1.0 \%$ and $10.0 \%$ drug-loaded microspheres respectively. Glycine is the smallest amino acid with a molar mass of $75 \mathrm{~g} / \mathrm{mol}$. The addition of glycine to the BSA in the copolymer matrix brought the melting point of the BSA/Gly microspheres down to $257.63^{\circ} \mathrm{C}$ for the blank. Interestingly, the addition of resveratrol drug moderated the melting of the $\mathrm{BSA} / \mathrm{Gly}$ microspheres and reduced it from $254.39^{\circ} \mathrm{C}$ in the $1.0 \%$ drug loading to $250.78^{\circ} \mathrm{C}$ in the $10.0 \%$ drug loading. BSA/Gly has been previously used to encapsulate bioactive nutraceuticals such as riboflavin with the spray-drying encapsulation method requiring inlet and outlet temperatures of $110^{\circ} \mathrm{C}$ and $74^{\circ} \mathrm{C}$ respectively without any adverse effect on the bioactivity of the drug ingredient [13]. These processing temperatures are very low compared to the melting point of the microspheres and the resveratrol drug compound. Since resveratrol has interesting properties and great potential for therapeutic application, this study is desirable. In line with this, De Cassia da Silva and co-workers subjected pure resveratrol in solid state that had no protection from encapsulation in polymer matrix to simultaneous thermogravimetry and differential scanning calorimetry [48]. The investigators reported that in both dry air and nitrogen atmospheres, the drug compound was thermally stable and decomposition was initiated only at up to $340^{\circ} \mathrm{C}$ without any losses. Under air atmosphere, mass loss of about $40 \%$ occurred only when the temperature was raised from the $240^{\circ} \mathrm{C}$ to $425^{\circ} \mathrm{C}$ [48]. Moreover, temperatures of around $250^{\circ} \mathrm{C}$ melting point of the microspheres are not the normal storage and body conditions that drug formulations are subjected to. Furthermore, both albumin and glycine have been used as stabilizers for proteins and other organic compounds under various formulation and processing conditions. Albumin is extensively used in blocking buffers for solid-base immunoassay because of its ability to stabilize the antigenic and functional regions of the adsorbed proteins. Glycine and glycine compounds have also been used extensively as protectants of a number of compounds against thermal inactivation [24]. It has been reported that the addition of glycine or glycylglycine to a formulation containing the apical membrane antigens 1 (AMA1) of malaria parasites prevented the loss of potency of the vaccine in clinical trials [21,23]. Glycine has been used to provide support during freeze-drying to prevent the macroscopic collapse of the final products in a number of formulations [25]. Resveratrol is subject to isomerization from the stable trans crystalline form to the metastable cis form by light and pH changes. However, isomerization of resveratrol has been reported to be partially reversible by heat treatments [33]. Also, the chemical instability has been reported to be overcome by encapsulation to protect the drug from environmental factors that promote chemical degradation during storage [49].

\begin{tabular}{|c|c|c|}
\hline Formulation Number & $\begin{array}{l}\text { Polymer Type } \\
(5 \% \mathrm{w} / \mathrm{v})\end{array}$ & $\begin{array}{c}\text { Melting Point } \\
\left({ }^{\circ} \mathrm{C}\right)\end{array}$ \\
\hline Blank 1 & BSA/GLY & $257.63 \pm 5.28$ \\
\hline Blank 2 & BSA & $296.7 \pm 3.56$ \\
\hline Blank 3 & Glycine & $253.37 \pm 1.15$ \\
\hline Microsphere Formulations & \multicolumn{2}{|c|}{$1.0 \%$ Drug Loading } \\
\hline $\mathrm{F} 1$ & BSA/GLY & $254.39 \pm 6.83$ \\
\hline F2 & BSA & $294.35 \pm 2.28$ \\
\hline F3 & Glycine & $253.75 \pm 1.53$ \\
\hline Microsphere Formulations & \multicolumn{2}{|c|}{$10.0 \%$ Drug Loading } \\
\hline F4 & BSA/GLY & $262.56 \pm 2.71$ \\
\hline F5 & BSA & $301.14 \pm 11.36$ \\
\hline F6 & Glycine & $250.78 \pm 1.13$ \\
\hline Resveratrol Drug & $\mathrm{N} / \mathrm{A}$ & $254 \pm 1.35$ \\
\hline
\end{tabular}

Table 3: The melting points of the resveratrol-loaded microspheres in the different polymer matrixes and at different drug loading. 


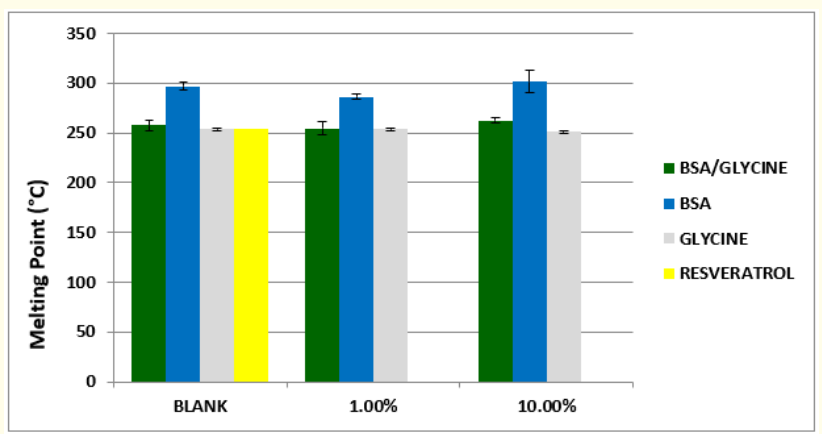

Figure 4: The melting points of the resveratrol-loaded microspheres in the different polymer matrixes and at different drug loading.

\section{Dissolution and drug release}

In vitro release or dissolution studies were performed to predict the pattern of resveratrol release from all the polymer matrixes. The studies were conducted on the $0.5 \%$ glutaraldehyde crosslinked microspheres with 1.0 and 10.0 percent drug loading. The standard curve for resveratrol is presented in figure 5. The curve in figure 5 a shows that above the concentration 70 micrograms per milliliter, there was no linear relationship observed between the increasing resveratrol concentration and absorbance. The standard curve used in this study is shown in figure $5 \mathrm{~b}$ and was drawn with concentration up to 70 microgram per milliliter that shown linearity. The $\mathrm{R}^{2}$ value of 0.9992 indicates a good correlation between the absorbance and concentration of the resveratrol.

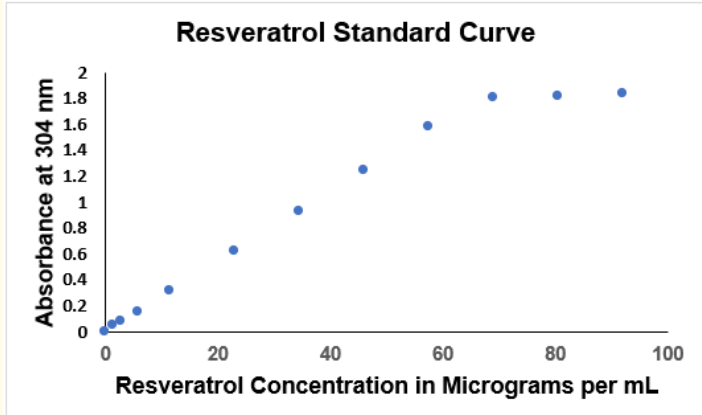

Figure 5A: Resveratrol concentration standard curve.

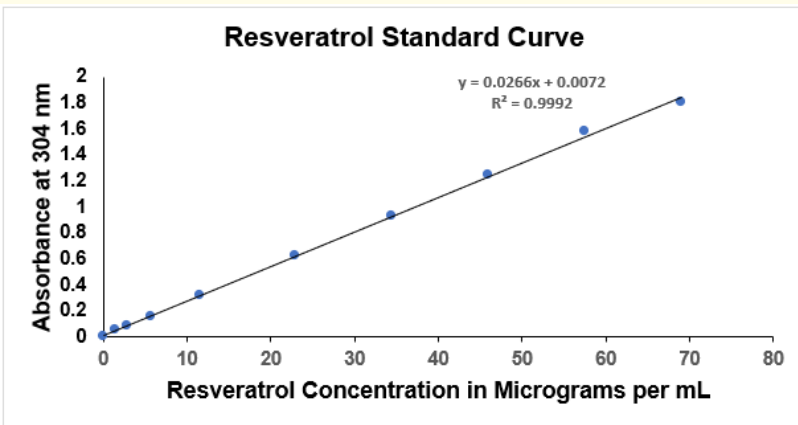

Figure 5b
The cumulative percent of the resveratrol released from the $0.5 \%$ glutaraldehyde crosslinked microspheres produced from all the six formulations is shown in figure 6 . The data shows an initial burst period for the first two hours followed by a sustained release in all the formulations. Characteristically the release profile was dependent on drug loading. The release was extended throughout the 24-hour study period in all the polymer matrixes of the $1.0 \%$ drug loaded microspheres. The initial burst release for this formulation lasted for up to four hours when over 50 percent of drug were released. By the end of the study period, the cumulative drug released were $94.13 \%, 91.64 \%$ and $92.85 \%$ for Glycine, BSA, and BSA/Glycine (copolymer) microspheres with $1.0 \%$ drug loading respectively. The percent release of drug was higher at all time points in the $10.0 \%$ drug loaded microspheres. The first two hours showed a burst release of about $70 \%$ of drug with almost $50 \%$ release in the first one hour alone. In addition, all the encapsulated drugs were released from the $10.0 \%$ drug-loaded BSA/Glycine and Glycine microspheres by the $12^{\text {th }}$ hour. In the $10.0 \%$ drug-loaded BSA microspheres, the release was extended for another six hours up to the $18^{\text {th }}$ hour before all the drugs were released. In all the formulations, more drug was released from the Glycine microspheres and the BSA microspheres had the longest release period of all the formulations. The data show that the addition of glycine moderated the release pattern of drugs from the BSA. This may be due to a less strong crosslinking of the glycine because of the small size and limited number of amine groups in the molecule as compared to the BSA [13]. It is worthy to note that both the product yield and drug release were higher in the $10.0 \%$ than the $1.0 \%$ drug-loaded microspheres. It is assumed that this drug loading level is effective in minimizing losses and getting most of the active drug released in the absorption windows of the intestines. The high levels of resveratrol detected in the release study can be attributed to two factors. First, resveratrol is lipophilic with a log P of 3.1, and thus highly immiscible and insoluble in water [50]. The high levels of released drug is an indication of the increased mixing, hydration and dissolution of the drug in the release buffer. Lui and his co-workers have reported that the aqueous solubility of resveratrol increases by about 35 times when encapsulated in alpha-lactalbumin as compared to the free drug [18]. Other investigators have reported similar findings when resveratrol is complexed with other polymers such as cyclodextrin, soya beans proteins, and pectin [50]. Secondly, resveratrol, a bioactive hydrophobic phenolic compound are able to be readily released from the hydrophilic protein polymer matrixes due to incompatibility of the compounds [18].

Evaluation of the mechanism of drug release from the formulation by higuchi plot analysis

The drug release data from the BSA/Glycine microspheres were fitted to different kinetic models. It was determined that the data fitted the Higuchi plot best thus suggesting a more diffusion and microsphere erosion release pattern. Higuchi in 1961 [12] developed an equation for the release of solid drug particles dispersed 
in homogeneous matrix dosage systems. The equation states that for a release based on a diffusion mechanism, the amount of drug released, $\mathrm{Q}$ is proportional to $\mathrm{A}$, the square root of the total amount of drug in unit volume of matrix, $\mathrm{D}$, the diffusion coefficient of the drug, $\mathrm{C}_{\mathrm{s}^{\prime}}$, the solubility of drug in the polymer matrix, and $\mathrm{t}$, the time of release. $\left(Q=2 A C_{s} t\right)^{1 / 2}$ for a given formulation, because $A, D$, and $\mathrm{C}_{\mathrm{s}}$ are all constant, for a dissolution and release based on diffusion, a plot of $\mathrm{Q}$ the amount or cumulative percentage of drug released by the square root of $t$, should follow a straight line [12].

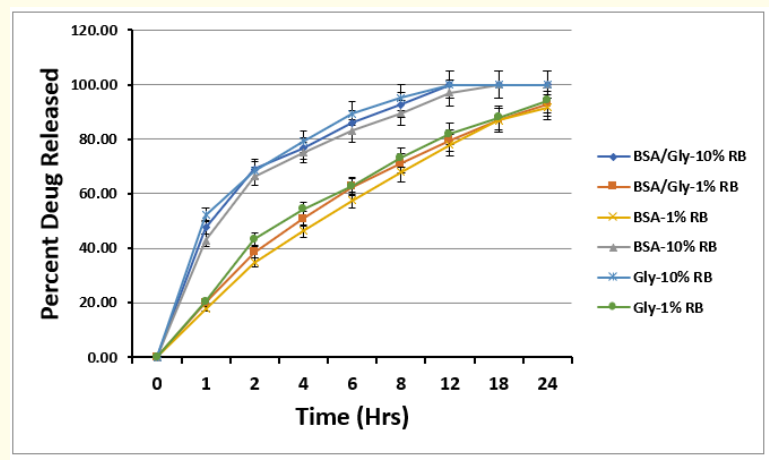

Figure 6: Cumulative release of resveratrol from the microsphere formulations in the different polymer matrixes.

Figure $7 \mathrm{a}$ and $7 \mathrm{~b}$ show the Higuchi plot analysis of the pattern of resveratrol release from the $1.0 \%$ and $10.0 \%$ BSA/Glycine matrix microspheres for the 24 hours. The graphs show that the pattern almost follow straight lines with $\mathrm{R}^{2}$ values of 0.9502 and 0.91 for the $1.0 \%$ and $10.0 \%$ drug-loaded microspheres respectively, thus indicating a primary diffusion controlled release for that time period. Even though all the $\mathrm{R}^{2}$ values were high, the 0.9502 for the $1.0 \%$ was more fitting of a straight line than the 0.91 for the $10.0 \%$ drug-loaded microspheres. This is expected of homogeneous matrix with less drug loading in the $1.0 \%$ than the $10.0 \%$ and is attributed in this case to a higher burst release from the microspheres with higher drug loading. It is reported that upon incubation of microparticles that follow diffusion controlled release in an aqueous medium, drug molecules located at or near the particle surface are dissolved by the penetrating liquid front and diffuse out into the surrounding medium within a short period of time (burst release). In the case of this study that happened with the first two hours of the $10.0 \%$ drug-loaded as compared to the four hours in the $1.0 \%$ drug-loaded microspheres.

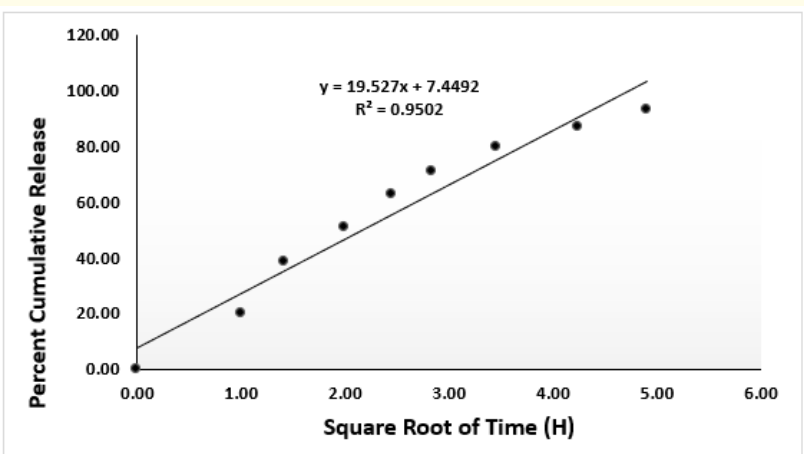

Figure 7a: Higuchi plot of the pattern of release of resveratrol from $1.0 \%$ drug-loaded BSA/Glycine microsphere.

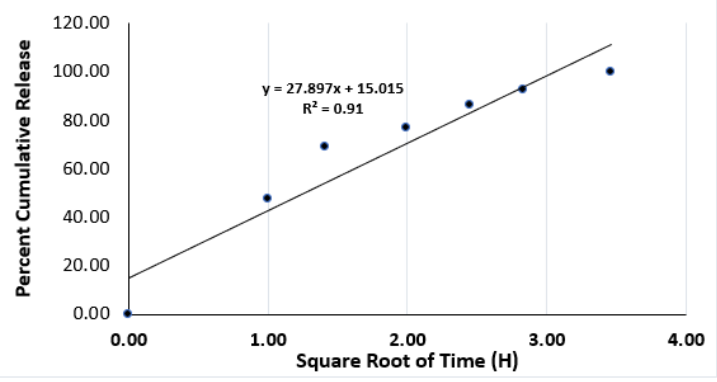

Figure 7b: Higuchi plot of the pattern of release of resveratrol from $10.0 \%$ drug-loaded BSA/Glycine microsphere.

\section{Release under oxygen stress and storage temperatures}

Resveratrol as a non-flavonoid compound is associated with the wine and food system. However, the utilization of resveratrol as a bioactive compound in the food industry is currently limited because of its sensitivity to some external agents such as air, light and oxidative enzymes. The food system formulations comprise large amounts of other components with reactive properties that induce stress and instability on the ingredients in least amount such as vitamins, flavonoid and nonflavonoids, as well as their delivery system such as microspheres $[48,50]$. As such, encapsulation is an effective approach to protect such bioactive functional nutraceuticals against such adverse conditions through their incorporation within the core of an encapsulating matrix system and improve their beneficial biological activities [18]. The study, therefore, was to evaluate the pattern of release of the resveratrol from the microspheres under oxygen stress and storage temperatures. These 
studies were to evaluate the release of resveratrol at the refrigerator and ambient temperatures of $4^{\circ} \mathrm{C}$ and $25^{\circ} \mathrm{C}$ when stored under varying oxygen stress released from hydrogen peroxide. Figure 8 show the results of the study. The data shows that there were more drugs released from the $10.0 \%$ than the $1.0 \%$ drug loading in all the release media including the blank water and at both temperatures (Figure 8a and 8c). It can be inferred that this is just as a result of higher drug loading. As shown in figure $8 \mathrm{~b}$ and $8 \mathrm{~d}$, the actual proportion of drug released as a percentage of the theoretical drug loading were higher in the $1.0 \%$ drug-loaded microspheres than the $10.0 \%$ in all the media including water. Furthermore, there were more drugs released at the $25^{\circ} \mathrm{C}$ storage temperature for all the formulations in all the media than the $4^{\circ} \mathrm{C}$. This should be attributed to the elevated temperature and the endothermic dissolution reaction associated with the increased energy in the warm media coupled with the higher release of oxygen to moderate the diffusion process and thus aid the dissolution. However, the a pat- tern of release was more discernable in the $10.0 \%$ drug-loading microspheres at the $4^{\circ} \mathrm{C}$ storage temperature. At this temperature the quantity and percentage of drug released reduced from the high of $10.0 \%$ hydrogen peroxide to the $5.0 \%$ with the least in the water medium. The differences in the quantities and percentages were significant $(\mathrm{p}<0.05)$. This pattern was not seen in the $1.0 \%$ drug-loaded microspheres at the $4^{\circ} \mathrm{C}$ and for all the formulations including the $10.0 \%$ drug-loaded microspheres as well as when the temperature was elevated to $25^{\circ} \mathrm{C}$. At the $25^{\circ} \mathrm{C}$ there was no significant difference in the drug release with increasing concentration of the hydrogen peroxide. The data shows that the water performed even better in releasing the drug than the $5 \%$ hydrogen period medium for the Glycine and BSA/Glycine microspheres at that temperature. This pattern is clearly shown in a comparative analysis of the data from the preferred the BSA/Glycine microspheres (Table 4 and figure 9). The study clearly shows that the increased oxygen stress and elevated storage temperature had effect on the release of resveratrol from the microspheres.

\begin{tabular}{|c|c|c|c|c|c|c|c|c|}
\hline \multirow{3}{*}{$\begin{array}{l}\text { Oxygen } \\
\text { Stress } \\
\text { Medium }\end{array}$} & \multicolumn{4}{|c|}{$1.0 \%$ Drug Loading (200 $\mu \mathrm{g})$} & \multicolumn{4}{|c|}{$10.0 \%$ Drug Loading (2000 $\mu \mathrm{g})$} \\
\hline & \multicolumn{2}{|c|}{$4^{\circ} \mathrm{C}$} & \multicolumn{2}{|c|}{$25^{\circ} \mathrm{C}$} & \multicolumn{2}{|c|}{$4^{\circ} \mathrm{C}$} & \multicolumn{2}{|c|}{$25^{\circ} \mathrm{C}$} \\
\hline & $\begin{array}{c}\text { Quantity } \\
(\mu g)\end{array}$ & Percent & $\begin{array}{c}\text { Quantity } \\
(\mu g)\end{array}$ & Percent & $\begin{array}{c}\text { Quantity } \\
(\mu \mathrm{g})\end{array}$ & Percent & $\begin{array}{c}\text { Quantity } \\
(\mu g)\end{array}$ & Percent \\
\hline $10 \% \mathrm{H}_{2} \mathrm{O}_{2}$ & 36.78 & 18.39 & 98.89 & 49.45 & 246.59 & 12.43 & 251.95 & 12.6 \\
\hline $5 \% \mathrm{H}_{2} \mathrm{O}_{2}$ & 27.29 & 13.65 & 74.56 & 37.31 & 162.58 & 8.13 & 246.29 & 12.31 \\
\hline $\mathrm{H}_{2} \mathrm{O}$ Only & 26.59 & 13.3 & 88.9 & 44.46 & 98.37 & 4.92 & 255.35 & 12.77 \\
\hline
\end{tabular}

Table 4: Resveratrol release from the microsphere formulations under varying levels of oxygen stress in different hydrogen peroxide concentrations at $4^{\circ} \mathrm{C}$ and $25^{\circ} \mathrm{C}$ storage temperature.

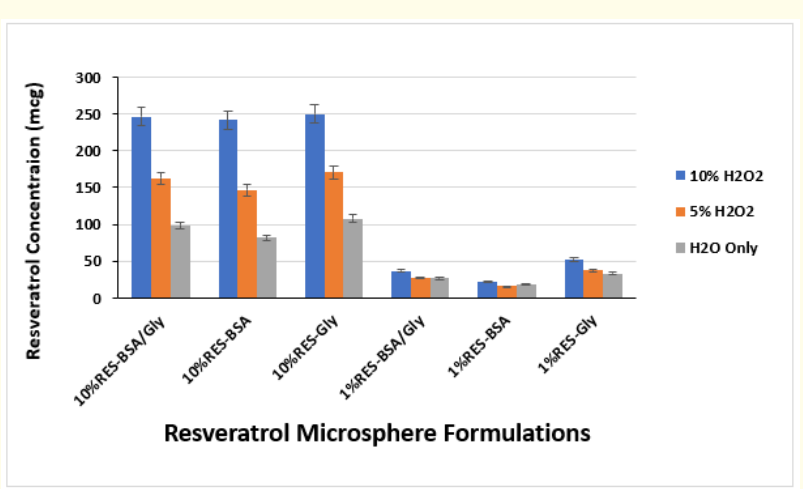

Figure 8a: Quantities of resveratrol released from the formulations at $4^{\circ} \mathrm{C}$ storage temperature.

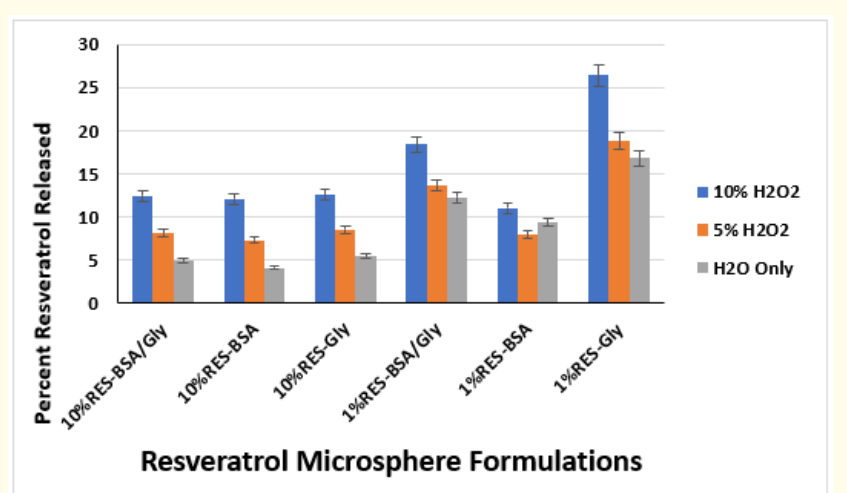

Figure 8b: Percent of original drug loading of resveratrol released from the microspheres at $4^{\circ} \mathrm{C}$ storage temperature. 


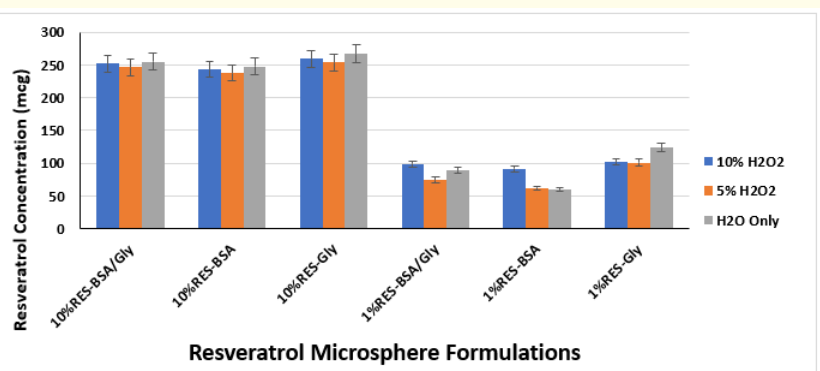

Figure 8c: Quantities of resveratrol released from the formulations at $25^{\circ} \mathrm{C}$ storage temperature.

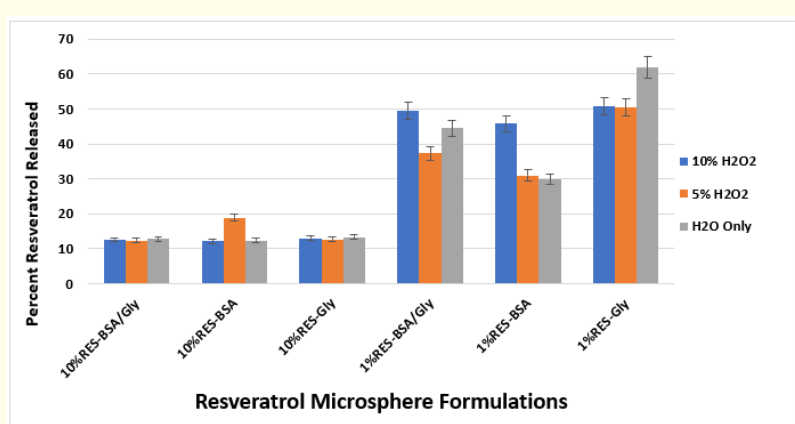

Figure 8d: Percent of the original drug loading of resveratrol released from the formulations at $25^{\circ} \mathrm{C}$ storage temperature.

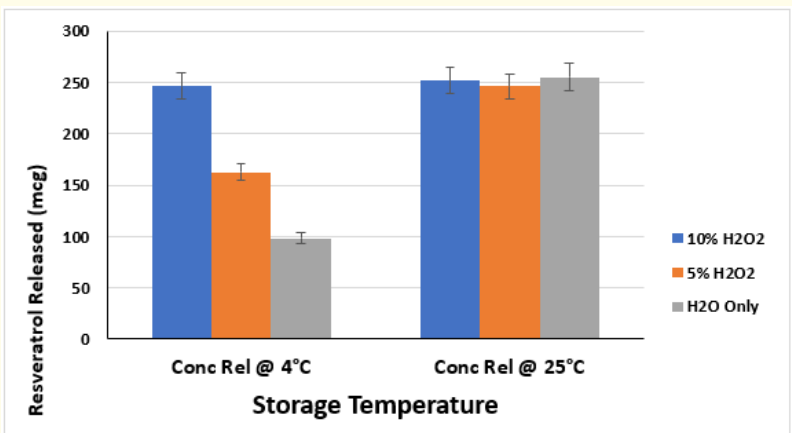

Figure 9a: Comparative release from the $10.0 \%$ drug-loaded BSA/ glycine microspheres at the at $4{ }^{\circ} \mathrm{C}$ and $25^{\circ} \mathrm{C}$ storage temperatures.

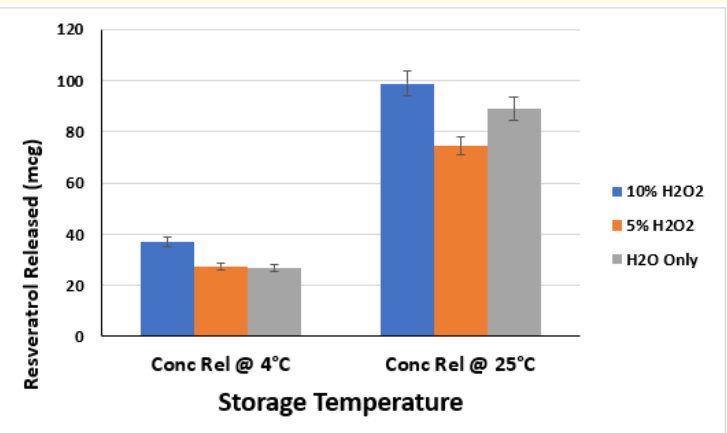

Figure 9b: Comparative release from the $1.0 \%$ drug-loaded BSA/ glycine microspheres at the at $4{ }^{\circ} \mathrm{C}$ and $25^{\circ} \mathrm{C}$ storage temperatures.

\section{Conclusion}

Overall, the study showed that the BSA/Glycine copolymer matrix produced microspheres with ideal characteristics to serve as oral delivery system for resveratrol. The study showed that the residual amount of glutaraldehyde after the crosslinking process and neutralization was below the toxic level for human. All the physicochemical characteristics of the BSA/Glycine microspheres were ideal for the delivery bioactive substance associated with the food and wine industry. The zeta potential, mean particle size, encapsulation efficiency, and in vitro drug release were within the required range for sustained oral delivery. The use of the polymer matrixes did not indicate any stability issue and conferred protection during storage and transport. It can be safely concluded that the BSA/ Glycine copolymers can be used to produce microspheres with the required specifications for the oral delivery of resveratrol. In this study, the BSA/Glycine with $5 \%$ weight-by-volume polymer concentration, at $0.5 \%$ volume-by-volume glutaraldehyde crosslinking with $10.0 \%$ resveratrol performed better than all the other formulations. It had very high encapsulation and drug release in addition to all the other physico-chemical characteristics.

\section{Declaration of Interest}

The authors report no conflict of interest. The authors alone are responsible for the content and writing of the paper.

\section{Bibliography}

1. Junk K-H., et al. "Resveratrol-loaded polymeric nanoparticles suppress glucose metabolism and tumor growth in vitro and in vivo". International Journal of Pharmaceutics 478 (2015): 251-257.

2. Chimento A., et al. "Progress to Improve Oral Bioavailability and Beneficial Effects of Resveratrol". International Journal of Molecular Science 20 (2019): 1381.

3. Peng H., et al. "Vanillin cross-linked chitosan microspheres for controlled release of resveratrol". Food Chemistry 121 (2010): 23-28.

4. Machado ND., et al. "Resveratrol Recent strategies in Resveratrol delivery systems”. ChemPlusChem 84 (2019): 951-973.

5. Santos A C., et al. "Nanotechnology-based formulations for resveratrol delivery: Effects on resveratrol in vivo bioavalibility and bioactivity". Colloids and Surfaces B: Biointerfaces 180 (2019): 127-140.

6. Andrade S., et al. "Resveratrol brain delivery for neurological disorders prevention and treatment". Frontiers in Pharmacology 9 (2018): 1261.

7. Brown V A., et al. "Repeat dose study of the cancer chemopreventive agent resveratrol in healthy volunteers: safety, pharmacokinetics, and effect on the insulin-like growth factor axis". Cancer Research, 70, 22 (2010): 9003-9011.

8. Davidov-Pardo G and McClememts D J. "Nutraceutical delivery systems: resveratrol encapsulation in grape see oil nanoemulsions formed by spontaneous emulsification". Food Chemistry 167 (2015): 205-212. 
9. Radko Y., et al. "Semi-preparative isolation of dihydroresveratrol-3-beta-d-glucuronide and four resveratrol conjugates from human urine after oral intake of resveratrol-containing dietary supplement". Journal of Chromatography B 930 (2013): 54-61.

10. Chung I-M., et al. "Resveratrol Nanoparticles: A promising therapeutic advancement over native resveratrol". Process 8 (2020): 458.

11. Zhao J., et al. "Improvement strategies for the oral bioavailability of poorly water-soluble flavonoids: An overview". International Journal of Pharmaceutics 570 (2019): 118642.

12. Yeboah KG and D'Souza M J. "Evaluation of Albumin microspheres as oral delivery system for Mycobacterium tuberculosis vaccines". Journal of Microencapsulation 26.2 (2009): 166-179.

13. Siddig A., et al. "Formulation and characterization of novel riboflavin containing albumin/glycine microspheres". International Journal of Pharmacy and Integrated Life Sciences 1.10 (2013): 1-25.

14. Galindo-Rodriquez S A., et al. "Comparative scale-up of three methods for producing ibuprofen-loaded nanoparticles". European Journal of Pharmaceutical Sciences 25 (2005): 357-367.

15. Sinha V R and Trehan A. "Biodegradable microspheres for protein delivery". Journal of Controlled Release 90 (2003): 261280.

16. Liu Y Gao., et al. "A-Lactalbumin and chitosan core-shell nanoparticles: resveratrol loading, protection, and antioxidant activity". Food and Function 11 (2020): 1525-1536.

17. Pantusa M., et al. "Stability of trans-Resveratrol associated with transport protein". Journal of Agriculture and Food Chemistry 62 (2014): 4384-4391.

18. Lui Y., et al. "Enhanced $\mathrm{pH}$ and thermal stability, solubility and antioxidant activity of resveratrol by nanocomplexation with alpha-lactalbumin". Food and Function 9 (2018): 4781-4790.

19. Thakkar H., et al. "Albumin microspheres as carriers for the antiarthritic drug celecoxib". AAPS PharmSciTech 6 (2005): E65-E73.

20. Bernard N G., et al. "Distribution and degradation of 1-125 albumin microspheres and technetium 99m sulphur colloid". Journal of Pharmaceutical Sciences, 15 (1980): 30-34.
21. Miles A P., et al. "Montanide ISA 720 vaccines: quality control of emulsions, stability of formulated antigens, and comparative immunogenicity of vaccine formulations". Vaccine 19 (2005): 2530-2539.

22. Ogienko A G., et al. "Large porous particles for respiratory drug delivery. Glycine-based formulations”. European Journal of Pharmaceutical Sciences 110 (2017): 148-156.

23. Zhu D., et al. "Long term stability of a recombinant Plasmodium falciparum AMA1 malaria vaccine adjuvanted with Montanide ISA 720 and stabilized with glycine". Vaccine 29 (2011): 3640-3645.

24. Papageorgiou G C and Murata N. "The unusually strong stabilizing effects of glycine betaine on the structure and function of the oxygen-evolving Photosystem II complex". Photosynthesis Research 44 (1995): 243-252.

25. Kasraian K., et al. "Characterization of the Sucrose/Glycine/ Water system by Differential Scanning Calorimetry and freeze-drying microscopy". Pharmaceutical Development and Technology 3.2 (1998): 233-239.

26. Adesunloye T A and Stach PE. "Effect of Glycine/Citric acid on the dissolution stability of hard gelatin capsules". Drug Development and Industrial Pharmacy 24.6 (1998): 493-500.

27. Silva C J S M., et al. "Chemical modification on proteins using glutaraldehyde". Food technology and Biotechnology 42.1 (2004): 51-56.

28. Migneualt I., et al. "Glutaraldehyde: behavior in aqueous solution, reaction with proteins, and application to enzyme crosslinking". Biotechniques 37.5 (2004): 790-802.

29. Furst $\mathrm{W}$ and Banerjee A. "Release of glutaraldehyde from an albumin-glutaraldehyde tissue adhesive causes significant in vitro and in vivo toxicity". Annals of Thoracic Surgery 79.5 (2005): 1522-1528.

30. Boratynski J and Zal T. "Colorimetric micro methods for glutaraldehyde determination by means of phenol and sulfuric acid or phenol and perchloric acid". Analytical Biochemistry 184.2 (1990): 259-262.

31. Jordan SL., et al. "Inactivation of glutaraldehyde by reaction with sodium bisulfite". Journal of Toxicology and Environmental Health 47.3 (1996): 299-309.

32. Trela B C and Waterhouse A L. "Resveratrol: Isomeric molar absorptivities and stability". Journal of Agricultural and Food Chemistry 44.5 (1996): 1253-1257. 
33. Camont L., et al. "Simple spectrophotometric assessment of the trans-/cis-resveratrol ratio in aqueous solutions". Analytica Chimica Acta 634 (2009): 121-128.

34. Ballantyne B and Jordan S L. "Toxicological, medical and industrial hygiene aspects of glutaraldehyde with particular reference to its biocidal use in cold sterilization procedures". Journal of Applied Toxicology 21.2 (2001): 131-151.

35. Ballantyne B and Myers RC. "The acute toxicity and primary irritancy of glutaraldehyde solutions". Veterinary and Human Toxicology 42.4 (2001): 193-202.

36. Galindo-Rodriquez S A., et al. "Polymeric nanoparticles for oral delivery of drugs and vaccines: A critical evaluation of in vivo studies". Critical Review in Therapeutics Drug Carrier Systems 22.5 (2005): 419-463.

37. Prinn K B., et al. "Statistical modeling of protein spray drying at the laboratory scale". AAPS PharmSciTech 3.1 (2002): 1-8.

38. Leach W T., et al. "Encapsulation of protein nanoparticles into uniform-sized microspheres formed in a spinning oil film". AAPS PharmSciTech 6 (2005): 605-617.

39. Cho A R., et al. "Preparation of chitosan-TPP microspheres as resveratrol carriers". Journal of Food Science 79.4 (2014): E568-E576.

40. Das S and Ng K Y. "Resveratrol loaded calcium-pectinate beads: effects of formulation parameters on drug release and bead characteristics". Journal of Pharmaceutical Sciences 99.2 (2010): 840-860.

41. Ko JA., et al. "Preparation and characterization of chitosan microparticles intended for controlled drug delivery". International Journal of Pharmaceutics 249 (2002): 165-174.

42. Muller BG., et al. "Albumin nanospheres as carriers for passive drug targeting: An optimized manufacturing technique". Pharmaceutical Research 13 (1996): 32-37.

43. Luftensteiner $\mathrm{CP}$ and Viernstein H. "Statistical experimental design based studies on placebo and mitoxantrone-loaded albumin microspheres". International Journal of Pharmaceutics 171 (1998): 87-99.

44. El-Mahdy M., et al. "Effects of preparation conditions on the monodispersity of albumin microspheres". Journal of Microencapsulation 15 (1998): 661-673.
45. Norris A and Sinko P. "Effect of size, surface charge, and hydrophobicity on the translocation of polystyrene microspheres through gastrointestinal mucin". Journal of Applied Polymer Science 63 (1998): 1481-1492.

46. Danaei M., et al. "Impact of particle size and Polydispersity Index on the clinical applications of lipidic nanocarrier systems". Pharmaceutics 10.57 (2018): 1-17.

47. Ahsan F., et al. "Targeting to macrophages: role of physicochemical properties of particulate carrier-liposomes and microspheres-on the phagocytosis by macrophages". Journal of Controlled Release 79 (2002): 29-40.

48. De Cassia da Silva R., et al. "Resveratrol: a thermoanalytical study". Food Chemistry 237 (2017): 561-565.

49. Davidov-Pardo G., et al. "Resveratrol encapsulation: Designing delivery systems to overcome solubility, stability and bioavailability issues". Trends in Food Science and Technology 38 (2014): 88-103.

50. Amri A., et al. "Administration of resveratrol: What formulation solutions to bioavailability limitations?" Journal of Controlled Release 158 (2012): 182-193.

\section{Assets from publication with us}

- Prompt Acknowledgement after receiving the article

- Thorough Double blinded peer review

- Rapid Publication

- Issue of Publication Certificate

- High visibility of your Published work

Website: $\underline{w w w}$.actascientific.com/

Submit Article: www.actascientific.com/submission.php

Email us: editor@actascientific.com

Contact us: +919182824667 